\title{
SENSOÂRS \\ Thermally Tunable Ultrasensitive Infrared Absorption Spectroscopy Platforms Based on Thin Phase-Change Films
}

\author{
Gokhan Bakan, ${ }^{* \dagger, \ddagger \odot}$ Sencer Ayas, ${ }^{\ddagger}, \#$ Erol Ozgur, ${ }^{\ddagger}$ Kemal Celebi, ${ }^{\ddagger}$ and Aykutlu Dana ${ }^{\ddagger}$ \\ ${ }^{\dagger}$ Department of Electrical and Electronics Engineering, Antalya International University, 07190 Antalya, Turkey \\ "UNAM Institute of Materials Science and Nanotechnology, Bilkent University, 06800 Ankara, Turkey
}

Supporting Information

ABSTRACT: The thermal tunability of the optical and electrical properties of phase-change materials has enabled the decades-old rewritable optical data storage and the recently commercialized phasechange memory devices. Recently, phase-change materials, in particular, $\mathrm{Ge}_{2} \mathrm{Sb}_{2} \mathrm{Te}_{5}$ (GST), have been considered for other thermally configurable photonics applications, such as active plasmonic surfaces. Here, we focus on nonplasmonic field enhancement and demonstrate the use of the phase-change materials in ultrasensitive infrared absorption spectroscopy platforms employing interference-based uniform field enhancement. The studied structures consist of patternless thin GST and metal films, enabling simple and large-area fabrication on
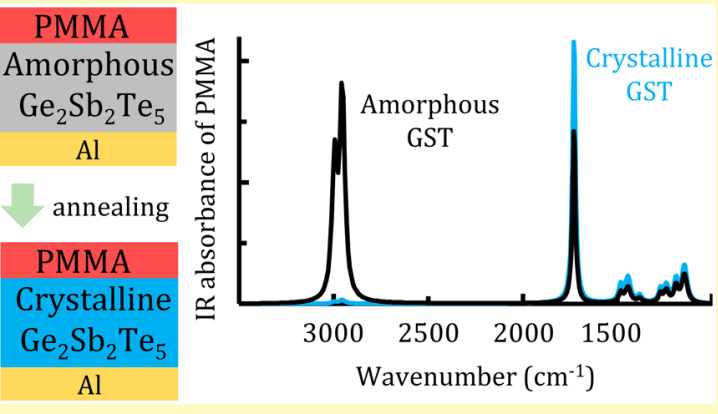
rigid and flexible substrates. Crystallization of the as-fabricated amorphous GST layer by annealing tunes (redshifts) the field-enhancement wavelength range. The surfaces are tested with ultrathin chemical and biological probe materials. The measured absorption signals are found to be comparable or superior to the values reported for the ultrasensitive infrared absorption spectroscopy platforms based on plasmonic field-enhancement.

KEYWORDS: GeSbTe, phase-change, infrared absorption spectroscopy, interference coatings, sensing

$\mathrm{P}$ hase-change materials exhibit reversible phase transitions between amorphous and crystalline states, accompanied by large changes in the materials' optical and electrical properties. The reversible changes in the material properties led to the development and the recent commercialization of the phasechange memory (PCM) devices. ${ }^{1}$ Long before the PCM devices had been widely studied, phase-change materials made rewritable optical data storage media possible in the 1990 s. $^{2}$ Optical data storage uses laser-induced heating to change the material's phase, hence the optical properties. More recently, the modulation of the optical properties of the phase change materials have been exploited for a variety of photonics applications, such as tunable light absorber/filter surfaces in the visible $^{3}$ and infrared ${ }^{4-6}$ regions using plasmonic structures, active chiral plasmonics, ${ }^{7}$ all-optical computing, ${ }^{8}$ integrated allphotonic nonvolatile memory, ${ }^{9}$ maskless photolithography, ${ }^{10}$ tunable bolometer pixels, ${ }^{11}$ color pixels, ${ }^{12,13}$ enhanced optical data storage, ${ }^{14}$ reversible surface phonon-polaritons resonators, ${ }^{15}$ and active control of surface plasmon waveguides. ${ }^{16}$ Here, we further extend this application list by demonstrating the use of GST thin films for thermally tunable, ultrasensitive infrared absorption spectroscopy platforms.

Infrared absorption spectroscopy is a widely used characterization method that reveals the molecular structure of materials through absorption of the incident infrared light at certain wavelengths. Such absorption wavelengths are determined by the molecular vibration modes, and the absorption magnitudes (signals) scale with the electric field intensity at the molecular vicinity and the volume of the probe material interacting with the infrared light. To enhance the absorption signal for a small amount of probe material, such as nanometer-thick films, attenuated total internal reflection (ATR) ${ }^{17}$ and grazing angle ${ }^{18}$ methods are commonly used. The ATR method requires the probe material making contact with a high-refractive-index crystal in which the infrared light travels and interacts with the material by multiple internal reflections. The grazing angle method exploits the field enhancement on a metal mirror at extreme angles of incidence. Hence, both methods require laborious sample preparations and special optical apparatus to send and collect infrared light, whereas it is also possible to achieve field enhancement by structural changes of the detection media, without requiring complex optical setups. In the past decade, developments in nanofabrication and plasmonics yielded extreme field-intensity enhancements (up to $10^{5}$-fold) on nanopatterned surfaces, enabling a new technique called surface enhanced infrared absorption spectroscopy (SEIRA). SEIRA has been utilized for detecting ultrathin probe materials such as poly(methyl methacrylate) (PMMA), ${ }^{19}$ monolayers of octadecanthiol (ODT), ${ }^{20,21}$ and protein molecules. $^{22,23}$ The field intensities on such surfaces, however, are enhanced only for a narrow spectral band that can only be tuned either by changing the design of the plasmonic

Received: September 20, 2016

Accepted: November 22, 2016

Published: November 22, 2016 
structures $^{23}$ or using electrostatically tunable materials like graphene. $^{24}$ Here, we use GST to demonstrate thermally tunable infrared absorption spectroscopy platforms based on the uniform field enhancement. It has been recently demonstrated that the uniform field-enhancement surfaces offer an easy and low-cost fabrication route for enhanced absorption in the infrared using thin metal films, ${ }^{25}$ graphene, ${ }^{26}$ and ultrathin chemical and biological films ${ }^{27}$ as the absorber layer.

To generate the uniform field enhancement, we fabricate surfaces that consist of two continuous layers: the dielectric film and the metal mirror. The incident and reflected rays from the dielectric-metal interface constructively interfere on the dielectric surface, when the wavelength $(\lambda)$ is equal to $\sim 4 n t$, where $n$ and $t$ are the refractive index and the thickness of the dielectric layer. The primary reflected ray from the airdielectric interface, however, is $180^{\circ}$ out of phase with respect to the incident ray, thus causing a partial destructive interference and reducing the electric field intensity at the air-dielectric interface (Figure S1). When a thin GST film is used as the dielectric layer, the field intensity enhancement factor is calculated as $\sim 3.6$, being $10 \%$ lower than the theoretical maximum of 4 that can be achieved when air is used as the dielectric layer. As for the metal mirror layer, $\mathrm{Al}$ is preferred over $\mathrm{Ag}$ and $\mathrm{Au}$ due to its abundance, low-cost and high reflectance in the infrared.

Optical simulations verify the effect of phase change and compare the GST/Al platforms to a simple uniform-field platform: $\mathrm{CaF}_{2}$ substrate with a field intensity enhancement factor of 0.7 on the substrate surface (Figure 1a). A 10-nmthick PMMA layer is used as the probe material for this study owing to its large number of absorption bands ranging from 3000 to $1000 \mathrm{~cm}^{-1}(\lambda=3.3$ to $10 \mu \mathrm{m}) . \mathrm{CaF}_{2}$ substrates are transparent in the infrared and can provide large absorption signals for large amount ( $\mu$ m-thick) of probe materials. ${ }^{28}$ For a 10-nm-thick PMMA film, however, the absorption signals are calculated to be less than $1 \%$. Specifically, the absorption bands at 2997 and $2952 \mathrm{~cm}^{-1}$ are observed with $0.14 \%$ and $0.18 \%$ magnitudes (Figure $1 \mathrm{~b}$ ). These absorption signals can be enhanced by a factor of 4.9 using amorphous GST (aGST)/Al surface with an aGST thickness of $200 \mathrm{~nm}$. However, the absorption signals for the higher wavelength bands (1732$1051 \mathrm{~cm}^{-1}$ ) are lower than those observed for the $\mathrm{CaF}_{2}$ substrate. Crystallizing the GST layer changes the optical properties of GST and as a result shifts the enhancement band to higher wavelengths and enhances the absorption at 1732 $\mathrm{cm}^{-1}$. The signal enhancement results show the correlation between the electric field intensity and the absorption signals (Figure 1c).

The electric field on the $\mathrm{CaF}_{2}$ substrate is determined by the interference of the incident ray and the reflected ray from the surface. Since the partial reflection is out of phase with respect to the incident light and no other in-phase secondary reflections are present, the total electric field intensity becomes smaller than the incident field intensity $\left(|E|^{2} /\left|E_{0}\right|^{2}=0.7\right)$. Despite the modest electric field intensities, $\mathrm{CaF}_{2}$ substrates are commonly used for infrared absorption spectroscopy due to the spatial and spectral uniformity of the electric field intensity on the surfaces (Figure 2a), whereas on aGST/Al surface, the field intensity enhancement factor is above unity for a wide range of wavelength $(2.7-5 \mu \mathrm{m})$. Furthermore, the field enhancement is not just limited to the surface, but extends hundreds of nanometers above (Figure 2b). The large extent of field (a)

(b)

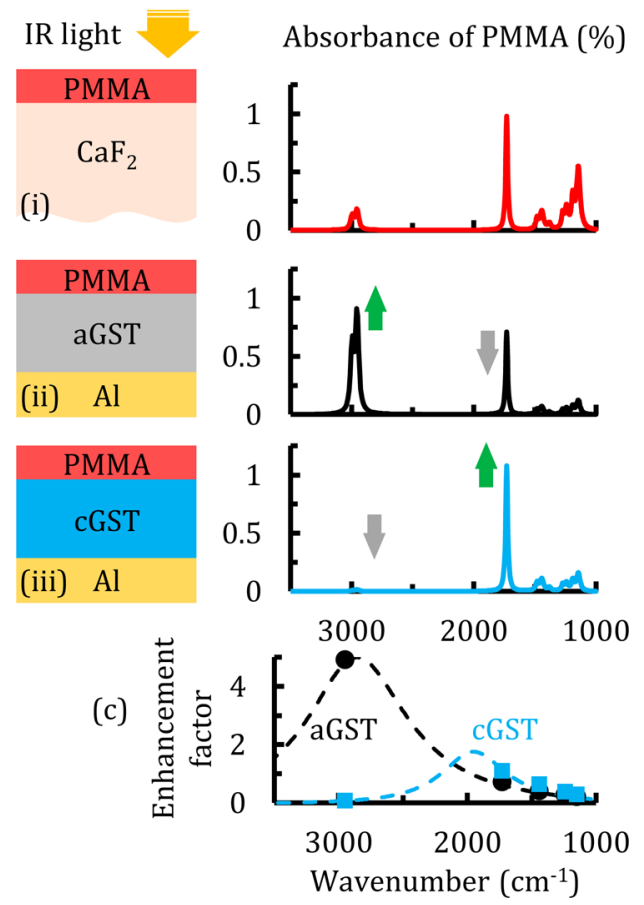

Figure 1. Comparison of the infrared absorption spectroscopy performances of $\mathrm{GST} / \mathrm{Al}$ platforms and $\mathrm{CaF}_{2}$ substrate. (a) Illustration of the cross sections of the sensing platforms: 10-nm-thick PMMA on (i) $\mathrm{CaF}_{2}$ substrate, (ii) aGST/Al, and (iii) cGST/Al. GST and Al thicknesses are 200 and $100 \mathrm{~nm}$, respectively. (b) Calculated PMMA absorption spectrum for each case. Upward and downward arrows highlight the enhanced and reduced signal intensities, respectively. (c) Dots: PMMA absorption signal intensities for aGST/Al and cGST/Al platforms scaled by the PMMA absorption signals for the $\mathrm{CaF}_{2}$ substrate. Major PMMA absorption bands at 2952, 1732, 1444, 1244 , and $1151 \mathrm{~cm}^{-1}$ are used for the plot. Dashed lines: Electric-fieldintensity $\left(|E|^{2}\right)$ on the PMMA layer for GST/Al platforms scaled by I $\mathrm{El}^{2}$ on the PMMA layer on the $\mathrm{CaF}_{2}$ substrate. PMMA's optical properties used for the optical simulations are shown in Figure S2.

enhancement offers greater absorption signals for a larger amount of probe materials (Figure S3), in contrast with the plasmonic surfaces on which the field enhancement typically decays within $100 \mathrm{~nm}$ above the surface. ${ }^{29}$ The field intensity enhancement band for crystalline GST (cGST)/Al surface shows a redshift to the $4.5-6 \mu \mathrm{m}$ range owing to the larger real refractive index of cGST $(n=6-7)$ in the infrared. The maximum enhancement factor, however, reduces to 1.8 due the nonzero extinction coefficient (Figure 2c). Figure S4 shows the optical properties of aGST and cGST used for the simulations.

The lossy nature of cGST leads to strong absorption of the IR light as observed on the measured reflection spectra of bare GST/Al surfaces (Figure 3). On the other hand, aGST is a lossless dielectric beyond $\lambda=1.5 \mu \mathrm{m}$, thus the absorption by the aGST/Al surface is weaker in the infrared. For wavelengths smaller than $1.5 \mu \mathrm{m}$, aGST is also a lossy dielectric which can generate bright colors when coated on metals as a result of spectrally selective strong absorption in the visible regime. ${ }^{13,14}$ The electric field enhancement bands shown in Figure 2 and the observed reflection minimums are closely related, as the field enhancement increases the absorption of the incident light by the lossy layers, i.e., cGST and metal. The absorption wavelength redshifts with increasing GST thickness and crystallization of the GST layer as shown by the measured 


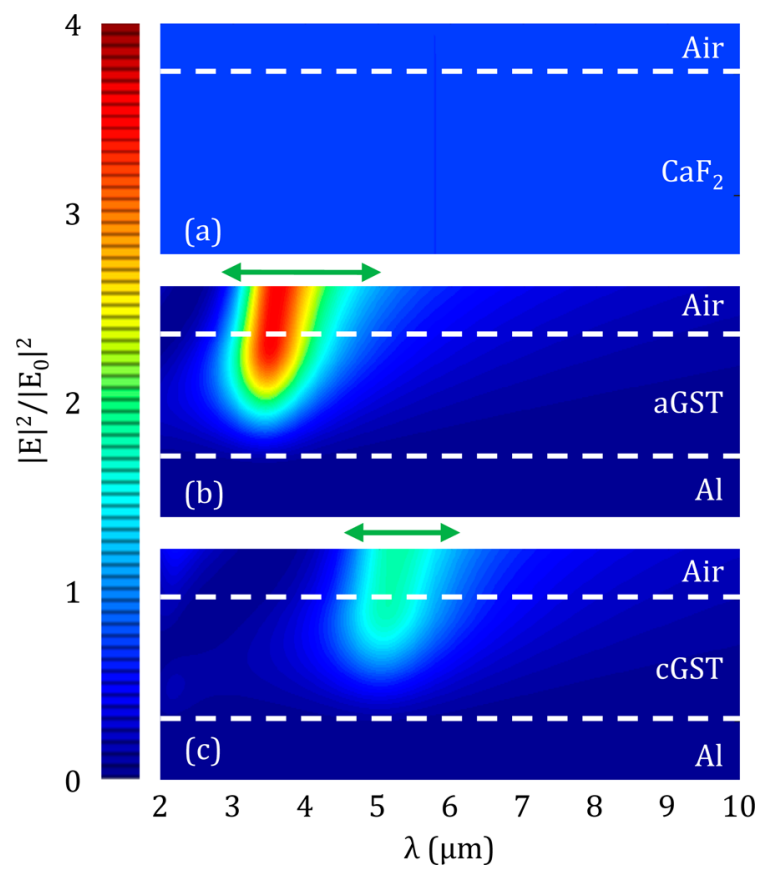

Figure 2. Simulated electric-field-intensities scaled by the incident field intensity on the cross sections of the sensing platforms. Boundaries between the layers are indicated with dashed lines. All the surfaces are covered with $10 \mathrm{~nm}$ PMMA layers. The electric field intensity enhancement bandwidths $\left(|E|^{2} /\left|E_{0}\right|^{2}>1\right)$ are shown with doubleheaded arrows for the GST/Al surfaces.

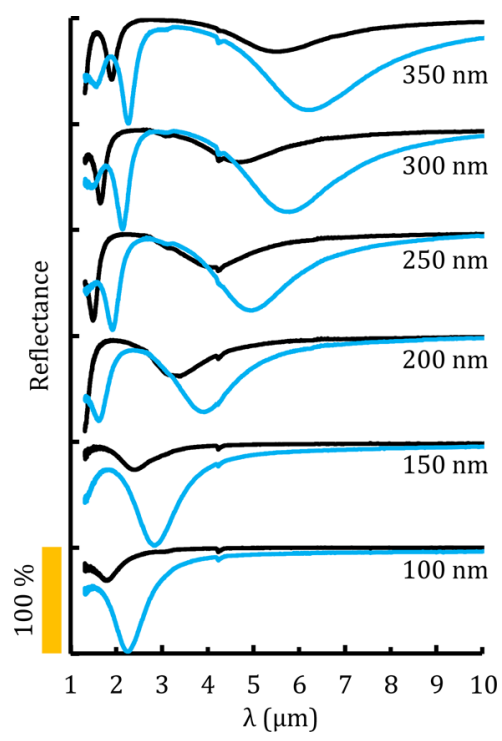

Figure 3. Infrared reflection spectra of bare aGST/Al (black) and cGST (blue) surfaces. GST thicknesses are shown next to each pair of curves. The curves are shifted along the $y$-axis for clarity.

reflection spectra (see Figure S5 for absorption wavelength vs GST thickness). The GST films are crystallized by annealing the surfaces beyond $150{ }^{\circ} \mathrm{C}$ on a hot plate for the reflection measurements and further sensing experiments. When laser annealing is used, amorphous to crystalline area ratio can also be gradually changed instead of a complete crystallization resulting in a mixed optical response (Figure S6). The reflection spectra of the bare GST/Al surfaces in Figure 3 show no parasitic vibrational absorption signals except low levels of absorption by $\mathrm{CO}_{2}$ and water vapor at $\sim 4.3 \mu \mathrm{m}$ $\left(\sim 2300 \mathrm{~cm}^{-1}\right)$ and $\sim 3 \mu \mathrm{m}\left(\sim 3300 \mathrm{~cm}^{-1}\right)$, respectively. The clear reflection spectra of the bare surfaces help in spotting the tiny changes on the reflection spectra due to the vibrational absorption of atop probe materials. The almost perfect absorption of the infrared light observed for the crystalline films also enables thickness-tunable thermal radiation (Figure S7).

The infrared absorption sensing performance of the GST surfaces are tested using 10-nm-thick PMMA layers. The GST thicknesses are chosen as 200 and $350 \mathrm{~nm}$ targeting the PMMA vibrational bands around 3000 and $1500 \mathrm{~cm}^{-1}$, respectively. The PMMA absorption bands are observed as narrow dips on the reflection spectra (Figure $4 \mathrm{a}$ ). The magnitudes of the

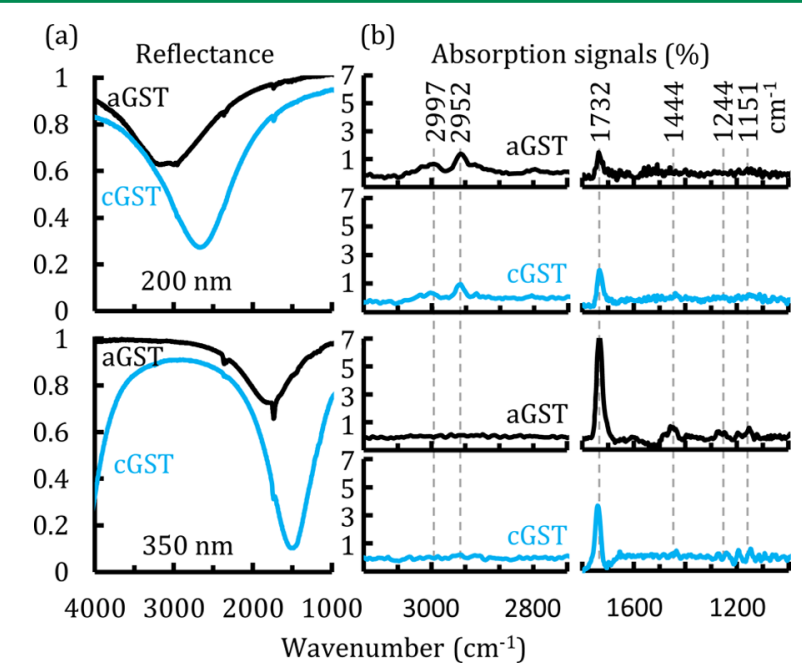

Figure 4. Infrared absorption spectroscopy of $10 \mathrm{~nm}$ PMMA films on GST/Al surfaces. (a) Reflection spectra of $10 \mathrm{~nm}$ PMMA coated aGST/Al and cGST/Al surfaces. 200 and $350 \mathrm{~nm}$ GST thicknesses are used to address PMMA's lower and higher wavelength absorption bands. (b) Absorption of the PMMA layers extracted from the reflection spectra in (a). The major PMMA absorption bands are highlighted with dashed lines.

PMMA absorption can be quantified after subtracting the backgrounds (Figure 4b). The background signals are generated by smoothing the reflection curves (Figure S8). Using such a method to generate the background signal eliminates the need for measurement of a reference sample. aGST $(200 \mathrm{~nm}) / \mathrm{Al}$ surface can sense the PMMA absorption bands at 2997, 2952, and $1732 \mathrm{~cm}^{-1}$. Crystallization of the GST layer, prior to coating the PMMA layer, lowers the absorption at 2997 and $2952 \mathrm{~cm}^{-1}$ and enhances the absorption at 1732 and $1444 \mathrm{~cm}^{-1}$ (Figure 4). The major PMMA band at 1732 $\mathrm{cm}^{-1}$ is observed with a signal intensity of $\sim 7 \%$ on aGST $(350$ $\mathrm{nm}) / \mathrm{Al}$ surface. This surface is particularly good at sensing all the vibrational bands between 1732 and $1151 \mathrm{~cm}^{-1}$. On the cGST $(350 \mathrm{~nm}) / \mathrm{Al}$ surface, although the signal intensity for $1732 \mathrm{~cm}^{-1}$ band drops to $3.5 \%$, the higher-wavelength absorption bands $\left(1192-754 \mathrm{~cm}^{-1}\right)$ appear as clear peaks. The enhanced absorption signals, especially for aGST/Al surface, are larger than the values reported for SIERA studies using plasmonic structures. ${ }^{19}$

The sensing performances of the surfaces are further tested with monolayers of octadecanthiol (ODT) and protein (bovine serum albumin, BSA) molecules. Such probe materials are typically used for benchmarking ultrasensitive SEIRA substrates. $^{20-23}$ ODT molecules are known to adhere well to $\mathrm{Ag}^{30}$ 
or $\mathrm{Au}^{21}$ surfaces. Therefore, GST/Al surfaces are first covered with Au nanoparticles formed by dewetting of 1.5-nm-thick $\mathrm{Au}$ film on the surfaces (Figure S9). A similar approach has been employed previously in refs 27,31 . The aGST thickness is chosen as $250 \mathrm{~nm}$ to adjust the field enhancement band close to the ODT absorption bands at 2849 and $2917 \mathrm{~cm}^{-1}$. The absorption signal for $2917 \mathrm{~cm}^{-1}$ band is found as $3 \%$ after background subtraction (Figure 5a). This value is in the range

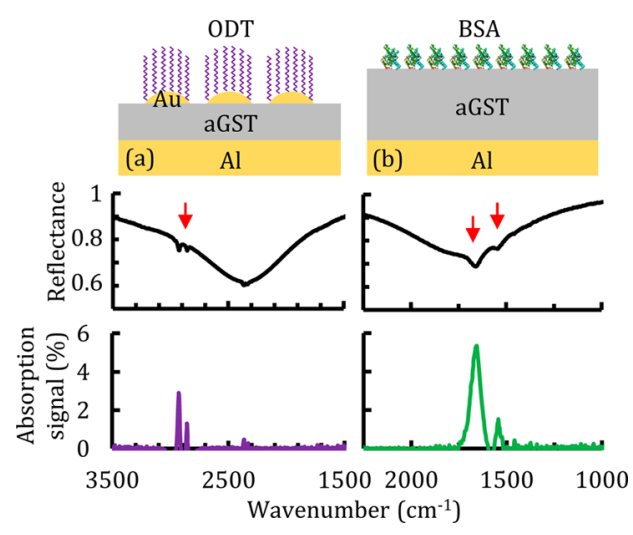

Figure 5. Monolayer sensing performance of aGST/Al platforms. Cross-section illustrations, reflection spectra, and absorption signals for (a) ODT and (b) BSA monolayers coated aGST/Al surfaces. aGST thicknesses are chosen as 250 and $350 \mathrm{~nm}$ for sensing ODT and BSA, respectively. The surface of 250 -nm-thick aGST is covered with $\mathrm{Au}$ nano islands to adhere ODT molecules to the surface. Arrows point at the ODT and BSA absorption bands.

of what is reported for plasmonic surfaces optimized for the ODT absorption bands. ${ }^{20,21}$ Since ODT does not show strong vibrational absorption bands at the higher wavelengths, shifting the enhancement band though crystallization of the GST layer is found disadvantageous (Figure S10). BSA's major absorption bands (Amide I and Amide II) are located at 1652 and 1531 $\mathrm{cm}^{-1}$, hence requiring a thicker aGST layer $(350 \mathrm{~nm})$ for absorption enhancement. The BSA thickness on aGST is extracted as $2-2.5 \mathrm{~nm}$ using spectroscopic visible ellipsometer measurements confirming the monolayer formation. The absorption signals for Amide I and Amide II bands are observed as $\sim 5.4 \%$ and $1.5 \%$ (Figure $5 \mathrm{~b}$ ). The large absorption signals, especially for Amide I band, are attributed to the good overlap of the field enhancement band with the BSA's absorption bands. The measured absorption signal for Amide I band is larger than the values (3-4\%) reported for the plasmonic surfaces. ${ }^{22,23}$ BSA sensing measurements are repeated using $\mathrm{Al}$ foils as the mirror layer and as well as the substrate (Figure S11). Despite the lower absorption signals, the results are promising for development of bendable, inexpensive, and disposable platforms using uniform field enhancement on GST covered flexible substrates.

\section{CONCLUSIONS}

In conclusion, we propose thin phase-changing GST films on Al mirrors as thermally tunable, ultrasensitive IR absorption spectroscopy platforms. The absorption enhancement is achieved by enhancing the electric field intensity by a factor of 3.6 on the surface. Crystallization of the GST layer redshifts the enhancement band owing to the higher refractive index of cGST while reducing the maximum field enhancement factor due to the lossy nature of cGST. The enhanced absorption signals are observed to be larger than most of the reports on surfaces using plasmonic field enhancement. The GST surfaces, especially in amorphous phase, sense the monolayers of ODT and BSA molecules with vibrational absorption signals comparable or larger than previous reports using plasmonic surfaces. The demonstrated surfaces have the potential for widespread usage for infrared absorption spectroscopy of ultrathin materials owing to easy, patternless, low-cost, and large-area fabrication of the surfaces, and also the ability to tune the field-enhancement band by phase change.

\section{ASSOCIATED CONTENT}

\section{Supporting Information}

The Supporting Information is available free of charge on the ACS Publications website at DOI: 10.1021/acssensors.6b00591.

Supplementary figures for reflected electric field magnitudes for an amorphous GST film on Al, optical properties of PMMA and GST films, IR camera images of crystalline GST on Al surfaces, measured reflected spectra for PMMA and ODT molecules, the measurement results using $\mathrm{Al}$ foils as the substrate and SEM images of $\mathrm{Au}$ nano islands. Experimental section describing the materials and methods used in this study. (PDF)

\section{AUTHOR INFORMATION}

\section{Corresponding Author}

*E-mail: gokhan.bakan@antalya.edu.tr.

ORCID

Gokhan Bakan: 0000-0001-8335-2439

\section{Present Address}

\#Bio-Acoustic-MEMS in Medicine (BAMM) Laboratory, Department of Radiology, Canary Center at Stanford for Cancer Early Detection, Stanford University School of Medicine, Palo Alto, California 94304, USA

\section{Notes}

The authors declare no competing financial interest.

\section{ACKNOWLEDGMENTS}

This work is partially supported by TUBITAK grant \#114E960 and EU FP7:People-IAPP NanoBacterPhageSERS.

\section{REFERENCES}

(1) Raoux, S.; Xiong, F.; Wuttig, M.; Pop, E. Phase change materials and phase change memory. MRS Bull. 2014, 39 (8), 703-710.

(2) Wuttig, M.; Yamada, N. Phase-change materials for rewriteable data storage. Nat. Mater. 2007, 6 (11), 824-832.

(3) Cao, T.; Wei, C.; Simpson, R. E.; Zhang, L.; Cryan, M. J. Broadband polarization-independent perfect absorber using a phasechange metamaterial at visible frequencies. Sci. Rep. 2014, 4, 3955.

(4) Michel, A.-K. U.; Zalden, P.; Chigrin, D. N.; Wuttig, M.; Lindenberg, A. M.; Taubner, T. Reversible optical switching of infrared antenna resonances with ultrathin phase-change layers using femtosecond laser pulses. ACS Photonics 2014, 1 (9), 833-839.

(5) Chen, Y.; Li, X.; Luo, X.; Maier, S. A.; Hong, M. Tunable nearinfrared plasmonic perfect absorber based on phase-change materials. Photonics Res. 2015, 3 (3), 54.

(6) Rudé, M.; Mkhitaryan, V.; Cetin, A. E.; Miller, T. A.; Carrilero, A.; Wall, S.; de Abajo, F. J. G.; Altug, H.; Pruneri, V. Ultrafast and Broadband Tuning of Resonant Optical Nanostructures Using PhaseChange Materials. Adv. Opt. Mater. 2016, 4 (7), 1060-1066. 
(7) Yin, X.; Schäferling, M.; Michel, A.-K. U.; Tittl, A.; Wuttig, M.; Taubner, T.; Giessen, H. Active Chiral Plasmonics. Nano Lett. 2015, 15 (7), 4255-4260.

(8) Wright, C. D.; Liu, Y.; Kohary, K. I.; Aziz, M. M.; Hicken, R. J. Arithmetic and Biologically-Inspired Computing Using Phase-Change Materials. Adv. Mater. 2011, 23 (30), 3408-3413.

(9) Ríos, C.; Stegmaier, M.; Hosseini, P.; Wang, D.; Scherer, T.; Wright, C. D.; Bhaskaran, H.; Pernice, W. H. P. Integrated all-photonic non-volatile multi-level memory. Nat. Photonics 2015, 9 (11), 725732.

(10) Shintani, T.; Anzai, Y.; Minemura, H.; Miyamoto, H.; Ushiyama, $\mathrm{J}$. Nanosize fabrication using etching of phase-change recording films. Appl. Phys. Lett. 2004, 85 (4), 639.

(11) Tittl, A.; Michel, A.-K. U.; Schäferling, M.; Yin, X.; Gholipour, B.; Cui, L.; Wuttig, M.; Taubner, T.; Neubrech, F.; Giessen, H. Plasmonic Absorbers: A Switchable Mid-Infrared Plasmonic Perfect Absorber with Multispectral Thermal Imaging Capability. Adv. Mater. 2015, 27 (31), 4526.

(12) Hosseini, P.; Wright, C. D.; Bhaskaran, H. An optoelectronic framework enabled by low-dimensional phase-change films. Nature 2014, 511 (7508), 206-211.

(13) Bakan, G.; Ayas, S.; Saidzoda, T.; Celebi, K.; Dana, A. Ultrathin phase-change coatings on metals for electrothermally-tunable colors. Appl. Phys. Lett. 2016, 109 (7), 71109.

(14) Schlich, F. F.; Zalden, P.; Lindenberg, A. M.; Spolenak, R. Color Switching with Enhanced Optical Contrast in Ultrathin Phase-Change Materials and Semiconductors Induced by Femtosecond Laser Pulses. ACS Photonics 2015, 2 (2), 178-182.

(15) Li, P.; Yang, X.; Maß, T. W. W.; Hanss, J.; Lewin, M.; Michel, A.-K. U.; Wuttig, M.; Taubner, T. Reversible optical switching of highly confined phonon-polaritons with an ultrathin phase-change material. Nat. Mater. 2016, 15, 870-875.

(16) Rudé, M.; Simpson, R. E.; Quidant, R.; Pruneri, V.; Renger, J. Active Control of Surface Plasmon Waveguides with a Phase Change Material. ACS Photonics 2015, 2 (6), 669-674.

(17) Kanik, M.; Aktas, O.; Sen, H. S.; Durgun, E.; Bayindir, M. Spontaneous High Piezoelectricity in Poly(vinylidene fluoride) Nanoribbons Produced by Iterative Thermal Size Reduction Technique. ACS Nano 2014, 8 (9), 9311-9323.

(18) Hassan, M.; Ilev, I. Grazing incidence angle based sensing approach integrated with fiber-optic Fourier transform infrared (FOFTIR) spectroscopy for remote and label-free detection of medical device contaminations. Rev. Sci. Instrum. 2014, 85 (10), 103108.

(19) Abb, M.; Wang, Y.; Papasimakis, N.; de Groot, C. H.; Muskens, O. L. Surface-Enhanced Infrared Spectroscopy Using Metal Oxide Plasmonic Antenna Arrays. Nano Lett. 2014, 14 (1), 346-352.

(20) Cubukcu, E.; Zhang, S.; Park, Y.-S.; Bartal, G.; Zhang, X. Split ring resonator sensors for infrared detection of single molecular monolayers. Appl. Phys. Lett. 2009, 95 (4), 43113.

(21) Huck, C.; Toma, A.; Neubrech, F.; Chirumamilla, M.; Vogt, J.; De Angelis, F.; Pucci, A. Gold Nanoantennas on a Pedestal for Plasmonic Enhancement in the Infrared. ACS Photonics 2015, 2 (4), 497-505.

(22) Cetin, A. E.; Etezadi, D.; Altug, H. Accessible Nearfields by Nanoantennas on Nanopedestals for Ultrasensitive Vibrational Spectroscopy. Adv. Opt. Mater. 2014, 2 (9), 866-872.

(23) Chen, K.; Dao, T. D.; Ishii, S.; Aono, M.; Nagao, T. Infrared Aluminum Metamaterial Perfect Absorbers for Plasmon-Enhanced Infrared Spectroscopy. Adv. Funct. Mater. 2015, 25 (42), 6637-6643.

(24) Rodrigo, D.; Limaj, O.; Janner, D.; Etezadi, D.; Garcia de Abajo, F. J.; Pruneri, V.; Altug, H. Mid-infrared plasmonic biosensing with graphene. Science 2015, 349 (6244), 165-168.

(25) Kocer, H.; Butun, S.; Li, Z.; Aydin, K. Reduced near-infrared absorption using ultra-thin lossy metals in Fabry-Perot cavities. Sci. Rep. 2015, 5, 8157.

(26) Zhu, L.; Liu, F.; Lin, H.; Hu, J.; Yu, Z.; Wang, X.; Fan, S. Angleselective perfect absorption with two-dimensional materials. Light: Sci. Appl. 2015, 5 (3), e16052.
(27) Ayas, S.; Bakan, G.; Ozgur, E.; Celebi, K.; Dana, A. Universal Infrared Absorption Spectroscopy Using Uniform Electromagnetic Enhancement. ACS Photonics 2016, 3 (3), 337-342.

(28) Moss, D.; Nabedryk, E.; Breton, J.; Mantele, W. Redox-linked conformational changes in proteins detected by a combination of infrared spectroscopy and protein electrochemistry. Evaluation of the technique with cytochrome c. Eur. J. Biochem. 1990, 187 (3), 565-572.

(29) Neubrech, F.; Beck, S.; Glaser, T.; Hentschel, M.; Giessen, H.; Pucci, A. Spatial extent of plasmonic enhancement of vibrational signals in the infrared. ACS Nano 2014, 8 (6), 6250-6258.

(30) Bukasov, R.; Shumaker-Parry, J. S. Silver Nanocrescents with Infrared Plasmonic Properties As Tunable Substrates for Surface Enhanced Infrared Absorption Spectroscopy. Anal. Chem. 2009, 81 (11), 4531-4535.

(31) Ayas, S.; Topal, A. E.; Cupallari, A.; Güner, H.; Bakan, G.; Dana, A. Exploiting Native $\mathrm{Al}_{2} \mathrm{O}_{3}$ for Multispectral Aluminum Plasmonics. ACS Photonics 2014, 1 (12), 1313-1321. 